Trauma Berufskrankh 2018 20 (Suppl 4):S251-S253 https://doi.org/10.1007/s10039-018-0372-0 Online publiziert: 4. Mai 2018

(c) Springer Medizin Verlag $\mathrm{GmbH}$, ein Teil von Springer Nature 2018

CrossMark

\section{Lenz}

Spitzenverband der gewerblichen Berufsgenossenschaften und der Unfallversicherungsträger der öffentlichen Hand, Deutsche Gesetzliche Unfallversicherung (DGUV), Berlin, Deutschland

\title{
Aktuelles zum Ärztevertrag und zur Unfall-Gebührenordnung (UV-GOÄ)
}

Zum Vertrag Ärzte/Unfallversicherungsträger [1] und zur Unfall-Gebührenordnung (UV-GÖ̈) [2] gibt es aktuelle Änderungen, die nachfolgend dargestellt werden.

\section{Beschlüsse der Ständigen Gebührenkommission nach $\$ 52$ Vertrag Ärzte/ Unfallversicherungsträger vom 22.08.2017}

Die Ständige Gebührenkommission nach $\$ 52$ Vertrag Ärzte/Unfallversicherungsträger hat durch die Beschlüsse vom 22.08.2017 [3] eine Anpassung der Gebühren der UV-GÖ̈ um insgesamt $18 \%$, verteilt über einen Zeitraum von 4 Jahren beschlossen. Am 01.10.2017 wurden die Gebühren bereits um $8 \%$ erhöht. Es folgen 3 weitere Erhöhungen im jährlichen Abstand von je 3\%. Zusammengerechnet sind das zwar nur $17 \%$, bezogen auf den Ausgangswert aber $18 \%$. Einige Leistungen, die in den letzten Jahren angepasst bzw. neu in die UV-GOÄ aufgenommen wurden, sind von der Anpassung ausgeschlossen (z. B. Gutachtengebühren).

Der Teil D „Anästhesieleitungen“ wurde völlig neu gefasst und neu bewertet. Hierfür gab es gute Gründe:

- Die Leistungsbeschreibungen entsprachen nicht mehr dem aktuellen Stand moderner Anästhesieleistungen.

- Anästhesieleistungen waren untervergütet. Sie wurden seinerzeit bei Anpassung der ambulanten Operationsleistungen nicht berücksichtigt.

\section{- Es gab zahlreiche Abrechnungs- streitigkeiten wegen unklarer $\mathrm{Be}$ - grifflichkeiten (z. B. Narkosedauer) und zur Abrechnungsfähigkeit von Nebenleistungen.}

Daher wurden im Vorgriff auf eine zu erwartende neue amtliche Gebührenordnung (GOÄ) die Leistungslegenden auf einen aktuellen Stand gebracht, und eine angemessene Vergütung wurde vereinbart. Für die wichtigsten Anästhesieverfahren, nämlich die Allgemeinanästhesie (Narkose) und die rückenmarksnahen und die intravenösen Regionalanästhesien, wurden in Abstimmung mit dem Berufsverband Deutscher Anästhesisten Leistungsbeschreibungen nach aktuellem Stand unter Einschluss fast aller in Betracht kommenden Nebenleistungen wie medikamentöse Prämedikation, EKG, Pulsoxymetrie usw. entwickelt und dafür Komplexgebühren kalkuliert. Maßgeblich für die Höhe der Gebühr ist die Zeit, die der Anästhesist aufwenden muss. Daher erfolgt die Abrechnung nach Zeittakten: $60 \mathrm{~min}$ sind immer zu vergüten. Danach sind Zuschläge für je 30 min abrechenbar. Die Narkosedauer das bezieht sich nur auf die Allgemeinanästhesie - wird über die Schnitt-/NahtZeit definiert mit einem Zeitzuschlag von $25 \mathrm{~min}$ davor und danach. In den $\mathrm{Zu}-$ schlagskatalog für ambulante Operationen neu aufgenommen wurden die Beobachtung und Betreuung während der Aufwach- und Erholungszeit bis zu $2 \mathrm{~h}$. Bisher gab es einen Zuschlag nur für mehr als 2 h. Dieser Zuschlag kann wahlweise vom Anästhesisten oder vom Operateur abgerechnet werden, wenn er diese Leis- tung übernimmt. Die Änderungen sollten $\mathrm{zu}$ einer erheblichen Erleichterung der Rechnungslegung und Rechnungsprüfung und zu einer deutlichen Reduzierung von Streitfällen führen. Anästhesieleistungen, die der Operateur selbst erbringen kann, wie z.B. die Armplexusoder Paravertebralanästhesie, die Kurznarkosen oder die Kaudalanästhesie, sind unverändert erhalten geblieben. Anders als die neuen Leistungen werden diese auch in die Gebührenanpassung von $18 \%$ einbezogen.

\section{Kostenübernahme für Tetanusimpfungen durch die Unfallversicherungsträger}

Eine Änderung wurde für die Kostenübernahme von Tetanusimpfungen nach Arbeitsunfällen beschlossen. Während bisher nur die Kosten für eine evtl. erforderliche Passivimmunisierung und/oder eine Aktivimmunisierung mit Monoimpfstoff übernommen wurden, werden künftig für eine nach der Empfehlung der Ständigen Impfkommission (STIKO) [4] notwendige Passivimmunisierung auch die Kosten für den Kombiimpfstoff (in der Regel Tetanus/Diphtherie/Pertussis) übernommen. Kosten für Folgeimpfungen zum Aufbau der Grundimmunisierung werden vom Unfallversicherungsträger nicht bezahlt, da kein Zusammenhang mit dem Arbeitsunfall besteht.

Eine Aktivimmunisierung ist - soweit kein ausreichender Impfschutz besteht bzw. der Impfschutz unbekannt ist - in der Regel bei sauberen, geringfügigen Wunden durchzuführen. Bei anderen 
Wunden (tiefen und/oder verschmutzten Wunden) kann zusätzlich eine Passivimmunisierung erforderlich sein. Einzelheiten sind den jeweiligen STIKO-Empfehlungen zu entnehmen.

\section{Neuer Vertrag Ärzte/ Unfallversicherungsträger}

Am 01.01.2018 ist ein neuer Vertrag zwischen der Deutschen Gesetzlichen Unfallversicherung (DGUV) und der Sozialversicherung für Landwirtschaft, Forsten und Gartenbau (SVLFG) einerseits und der Kassenärztlichen Bundesvereinigung (KBV) andererseits (Vertrag Ärzte/Unfallversicherungsträger) in Kraft getreten. Neben redaktionellen Korrekturen enthält der Vertrag die Einführung einer bundesweiten Clearingstelle für Gebührenstreitigkeiten zwischen Ärzten und Unfallversicherungsträgern sowie die Einführung neuer bzw. geänderter Arztvordrucke ab 01.07.2018.

\section{Einrichtung einer bundesweiten Clearingstelle}

Die bundesweite Clearingstelle ersetzt die bisherigen Schlichtungsstellen auf Landesebene mit veränderter Aufgabenstellung. Die Geschäftsführung der neuen Clearingstelle liegt in jährlichem Wechsel bei der KBV und bei der DGUV. Sie ist mit je 2 Vertretern der KBV und der Unfallversicherungsträger besetzt. Clearinganträge der Ärzte sind mit einer schriftlichen Problemdarstellung und anonymisierten Unterlagen stets an die $\mathrm{KBV} \mathrm{zu}$ richten, und zwar möglichst elektronisch an clearingstelle-unfallversicherung@kbv.de. Dort erfolgt eine Vorprüfung. Hat der Antrag keine Aussicht auf Erfolg, erhält der Arzt eine begründete Ablehnung. Anträge mit Erfolgsaussicht werden in der Clearingstelle beraten. Über das Ergebnis werden die Beteiligten schriftlich informiert. Ein entsprechendes Verfahren gilt aufseiten der Unfallversicherungsträger. Der Rechtsweg wird durch die Entscheidung der Clearingstelle nicht verschlossen.

Trauma Berufskrankh 2018 - 20 (Suppl 4):S251-S253

https://doi.org/10.1007/s10039-018-0372-0

(c) Springer Medizin Verlag GmbH, ein Teil von Springer Nature 2018

\section{O. Lenz}

\section{Aktuelles zum Ärztevertrag und zur Unfall-Gebührenordnung (UV-GOÄ)}

\section{Zusammenfassung}

Zum Vertrag Ärzte/Unfallversicherungsträger und zur Unfall-Gebührenordnung (UV-GOÄ) gibt es aktuelle Änderungen. So hat die Ständige Gebührenkommission nach § 52 Vertrag Ärzte/Unfallversicherungsträger eine lineare Anpassung der Gebühren der UV-GOÄ von insgesamt $18 \%$, verteilt über einen Zeitraum von 4 Jahren, sowie eine Neufassung des Teils D "Anästhesieleistungen" beschlossen. Die Kosten für Tetanusimpfungen werden künftig auch bei Verwendung von „Kombiimpfstoffen“ im Rahmen der Empfehlungen der Ständigen Impfkommission (STIKO) vollständig übernommen. Mit dem zum
01.01.2018 in Kraft getretenen neuen Vertrag Ärzte/Unfallversicherungsträger wird eine bundesweite Clearingstelle für Gebührenstreitigkeiten installiert. Außerdem gibt es Verbesserungen bei den Arztvordrucken. Die weitere Entwicklung der UV-GOÄ ist abhängig davon, ob und wann der Verordnungsgeber eine neue amtliche Gebührenordnung (GÖ̈) verabschiedet.

Schlüsselwörter

Arztvordrucke · Clearingstelle - Gebührenanpassung · Ständige Gebührenkommission . Tetanusimpfung

\section{Current aspects on physician contracts and on accident insurance underwriters remuneration regulations for physicians (UV-GOÄ)}

\section{Abstract}

There are current amendments to the contract physicians/accident insurance underwriters and accident remuneration regulations (UVGOÄ). The Standing Remuneration Committee has decided on a linear adaptation of the charges of the UV-GOÄ from a total of $18 \%$, distributed over a period of 4 years and a revison of Part D "Anesthesia services", according to $\$ 52$ contract physicians/accident insurance underwriter. The charges for tetanus vaccinations will in the future be completely taken over even for the use of "combi-vaccines" within the framework of the recommendations by the Standing Vaccination Committee (STIKO). With the new contract physicians/accident insurance underwriter, which came into force on 1 January 2018, a nationwide clearing site for remuneration disputes has been installed. In addition, there are improvements in the physician forms. The further development of the UV-GOÄ is dependent on whether and when the regulatory body passes a new official set of remuneration regulations (GOÄ).

\section{Keywords}

Physician forms - Clearing site · Remuneration adaptation - Standing Remuneration Committee $\cdot$ Tetanus vaccination

\section{Änderungen bei den Arztvordrucken}

Zum 01.01.2018 ist der Überweisungsvordruck ÜV entfallen. Die Gebührenposition (Nr. 145 UV-GOÄ) bleibt aber bestehen, sodass die Gebühr für die Überweisung nach wie vor abgerechnet werden kann.

Mit Wirkung ab 01.07.2018 gibt es weitere Änderungen bei den Arztvordrucken. Der Durchgangsarztbericht wurde redaktionell überarbeitet. Die Angabe der Klassifikation der Arbeitsgemeinschaft für Osteosynthesefragen (AO-
Klassifikation) wird - soweit es sich um eine Fraktur handelt - zur Pflichtangabe. Das ICD 10-Feld bleibt freiwillig. Die Diagnose ist immer als "Freitext" anzugeben. Der Klartext des ICD 10Schlüssels reicht nicht aus. Das Feld zur Einleitung der Heilbehandlung wurde klarer gestaltet, sodass Fehlangaben weitgehend ausgeschlossen sind.

Weiterhin entfallen der „Nachschaubericht" und der "Zwischenbericht bei besonderer Heilbehandlung". Beide Berichte werden durch den neuen „Verlaufsbericht" ersetzt. Dieser ist so gestaltet, dass er sowohl als Heilverfahrensbe- 
richt - auch bei allgemeiner Heilbehandlung - als auch als Bericht über Nachschauuntersuchungen verwendet werden kann. Anders als der bisherige „Zwischenberichtbeibesonderer Heilbehandlung“ sieht er auch die Überleitung von der allgemeinen in die besondere Heilbehandlung vor.

Neu eingeführt wird der Ergänzungsbericht „Schulter“, der ab 01.07.2018 bei strukturellen Schulterverletzungen $\mathrm{zu}$ erstatten ist. Dieser sowie der unverändert gebliebene Ergänzungsbericht „Knie“ werden ab 01.07.2018 mit 25,00 € vergütet.

\section{Ausblick für die Weiter- entwicklung der UV-GOÄ}

Wie geht es weiter mit der Entwicklung der UV-GOÄ? Die Leistungslegenden von amtlicher Gebührenordnung (GOÄ) und UV-GOÄ sind zweifellos nicht mehr aktuell, um nicht zu sagen veraltet und daher dringend überarbeitungsbedürftig. Ausnahme ist das Kapitel Anästhesieleistungen in der UV-GOÄ. Im letzten Beschluss der Gebührenkommission vom August 2017 ist vereinbart, auf die neue amtliche GOÄ zu warten und dann ggf. die Teile nach und nach in die UV-GOÄ zu überführen. Fraglich ist, wann mit einer neuen GOÄ - die seit Langem angekündigt wird - zu rechnen ist. Im Koalitionsvertrag ist dazu Folgendes zu lesen:

„Sowohl die ambulante Honorarordnung in der Gesetzlichen Krankenversicherung (EBM), als auch die Gebührenordnung der Privaten Krankenversicherung (GOÄ) müssen reformiert werden. Deshalb wollen wir ein modernes Vergütungssystem schaffen, das den Versorgungsbedarf der Bevölkerung und den Stand des medizinischen Fortschritts abbildet. Dies bedarf einer sorgfältigen Vorbereitung. Die Bundesregierung wird dazu auf Vorschlag des Bundesgesundheitsministeriums eine wissenschaftliche Kommission einsetzen, die bis Ende 2019 unter Berücksichtigung aller hiermit zusammenhängenden medizinischen, rechtlichen und wirtschaftlichen Fragen Vorschläge vorlegt. Ob diese Vorschläge umgesetzt werden, wird danach entschieden.“
Unter diesen Umständen dürfte eine neue GOÄ vorerst nicht zu erwarten sein.

\section{Fazit für die Praxis}

\section{- Die Gebühren der UV-GOÄ erhöhen sich in den nächsten 3 Jahren jeweils zum 01. Oktober um je $3 \%$. \\ - Für Anästhesieleistungen gelten seit 01.10.2017 neue Leistungslegenden und Gebühren. \\ - Tetanusimpfungen nach Arbeitsun- fällen werden von den Unfallversiche- rungsträgern auch bei Verwendung von „Kombiimpfstoffen“ vollständig übernommen. \\ - Seit 01.01.2018 können sich Ärzte und Unfallversicherungsträger bei Abrechnungsstreitigkeiten an eine bundesweite Clearingstelle wenden. \\ - Ab 01.07.2018 wird der „Nach- schaubericht" durch den neuen „Verlaufsbericht" ersetzt.}

\section{Korrespondenzadresse}

\section{Lenz}

Spitzenverband der gewerblichen Berufsgenossenschaften und der Unfallversicherungsträger der öffentlichen Hand, Deutsche Gesetzliche Unfallversicherung (DGUV) Glinkastr. 40, 10007 Berlin, Deutschland otmar.lenz@dguv.de

\section{Einhaltung ethischer Richtlinien}

Interessenkonflikt. O. Lenz gibt an, dass kein Interessenkonflikt besteht.

Dieser Beitrag beinhaltet keine vom Autor durchgeführten Studien an Menschen oder Tieren.

The supplement containing this article is not sponsored by industry.

\section{Literatur}

1. Vertrag gem. § 34 Abs. 3 SGB VII zwischen der Deutschen Gesetzlichen Unfallversicherung e. V. (DGUV), Berlin, der Sozialversicherung für Landwirtschaft, Forsten und Gartenbau als landwirtschaftliche Berufsgenossenschaft (SVLFG), Kassel, einerseits und der Kassenärztlichen Bundesvereinigung, K.d.ö.R., (KBV) Berlin, andererseits über die Durchführung der Heilbehandlung, die Vergütung der Ärzte sowie die Art und Weise der Abrechnung der ärztlichen Leistungen (Vertrag Ärzte/Unfallversicherungsträger) Stand: 1. Januar 2018 (http://www.dguv.de/medien/inhalt/reha_ leistung/verguetung/aerzte.pdf)
2. UV - GOÄ (2017) (Euro - €) Gebührenordnung für Ärzte, Gesetzliche Unfallversicherung, mit Krankenhaus-Nebenkostentarif. http://www.dguv. de/medien/inhalt/reha_leistung/verguetung/uvgoae.pdf.Zugegriffen: 27.04.2018

3. Beschlüsse der Ständigen Gebührenkommission nach §52 Vertrag Ärzte/Unfallversicherungsträger vom 22. Aug. 2017 (http://www.dguv.de/medien/ inhalt/reha_leistung/verguetung/beschluss.pdf). Zugegriffen: 27.04 .2018

4. Robert Koch Institut (2017) Empfehlungen der Ständigen Impfkommission am Robert Koch Institut - 2017/2018. Epidemiologisches Bulletin, Nr. 34 